\title{
An Evolutionary Technique for Multicriterial Optimization Based on Endocrine Paradigm
}

\author{
Corina Rotar \\ “1 Decembrie 1918” University, Computer Science Department, Alba Iulia, Romania \\ crotar@uab.ro
}

\begin{abstract}
Many evolutionary algorithms have been lately developed for solving multiobjective problems, appealing or not to the Pareto optimality concept. Although, the evolutionary techniques for multiobjective optimization confront with several issues as: elitism, diversity of the population, or efficient settings for the specific parameters of the algorithm. In this paper, we propose a new evolutionary technique, which is inspired by the behavior of the endocrine system and uses the Pareto non-dominance concept. Therefore, the members of the population aren't called chromosomes anymore, but hormones and, even if they evolve according to the genetic principles, a supplementary mechanism based on the endocrine paradigm is connected with standard approach to deal with multiobjective optimization problems. Moreover, the proposed algorithm, in order to maintain the diversity of the population, uses a specific scheme of fitness sharing, eliminating the inconvenient of defining an appropriate value of sharing factor.
\end{abstract}

\section{Introduction}

Multiobjective optimization occupies a large area in technical literature. The optimization problems became difficult in more than a single objective's cases, so, the optimum concept is reformulated accordingly. Vilfredo Pareto offers the most common definition of optimum in multiobjective optimization (1896). The evolutionary techniques, which make use of Pareto concept of optimality, are so called Pareto-based approaches and were firstly suggested by Goldberg (1989). Since then, many Paretobased evolutionary techniques have been developed for multiobjective optimization problems.

Our paper proposes a new evolutionary algorithm for multiobjective optimization based on endocrine paradigm. The evolutionary algorithms for multiobjective optimization deal with specific issues as fitness assignment, diversity preservation or elitism. We also suggest in our paper a new paradigm, which could be a fruitful source of inspiration for evolutionary computation: endocrine paradigm. The hormonal system proves itself as a complex, semi-independent and adaptive system - qualities that could be exploited in the evolutionary techniques. 


\section{Corina Rotar}

\section{Definitions}

Formally, multiobjective optimization problem is given as follows. Considering $k$ criteria (1), $m$ inequality constrains $(\mathbf{2})$, and $p$ equality constrains $(\mathbf{3})$, we are interested in finding the decision variable vector $\overline{x^{*}}=\left(x_{1}^{*}, x_{2}^{*}, \ldots, x_{n}^{*}\right)$ from the domain $D, D \subset R^{n}$, which optimizes the vector function (1), and satisfies the constrains (2) and (3):

$$
\begin{gathered}
F(\bar{x})=\bar{f}(\bar{x})=\left(f_{1}(\bar{x}), f_{2}(\bar{x}), \ldots, f_{k}(\bar{x})\right) \\
g_{i}(\bar{x}) \geq 0, i=1,2, \ldots, m \\
h_{i}(\bar{x})=0, i=1,2, \ldots, p
\end{gathered}
$$

Since, we hardly ever detect a real-world situation where all the objective functions have the same optimum in the search space $D$, the concept of optimality should have been revised in the multiobjective optimization's context.

Vilfredo Pareto formulated the most common concept of optimality for multicriterial optimization problems. In order to estimate the quality of a particular element from the search space, a relation of dominance was established. We consider a $k$ objective minimization problem and a search domain $D \subset R^{n}$.

Definition 1: The vector $a=\left(a_{1}, a_{2}, \ldots, a_{k}\right)$ dominates the vector $b=\left(b_{1}, b_{2}, \ldots, b_{k}\right)$, if and only if the following statement if verified:

$$
\left(\forall i \in\{1,2, \ldots, k\}: a_{i} \leq b_{i}\right) \wedge\left(\exists i \in\{1,2, \ldots, k\}: a_{i}<b_{i}\right)
$$

Definition 2: The solution $x \in D$ is weakly non-dominated, if:

$$
\neg \exists y \in D \text { for which } \forall i \in\{1,2, \ldots, k\}: b_{i}<a_{i}
$$

where $f(y)=\left(b_{1}, b_{2}, \ldots, b_{k}\right)$ and $f(x)=\left(a_{1}, a_{2}, \ldots, a_{k}\right)$.

Definition 3: The solution $x \in D$ is strongly non-dominated, if:

$$
\neg \exists y \in D \text { for which }\left(\forall i \in\{1,2, \ldots, k\}: b_{i} \leq a_{i}\right) \wedge\left(\exists i \in\{1,2, \ldots, k\}: b_{i}<a_{i}\right)
$$

where $f(y)=\left(b_{1}, b_{2}, \ldots, b_{k}\right)$ and $f(x)=\left(a_{1}, a_{2}, \ldots, a_{k}\right)$.

Definition 4: The solution $x \in D$ is Pareto optimal if and only if the next assertion is verified:

$$
\neg \exists y \in D \text { for which } f(y)=\left(b_{1}, b_{2}, \ldots, b_{k}\right) \text { dominates } f(x)=\left(a_{1}, a_{2}, \ldots, a_{k}\right)
$$

Pareto front consists of the values of the objectives corresponding to the nondominated vectors found. 
An Evolutionary Technique for Multicriterial Optimization Based on Endocrine Paradigm 3

\section{Endocrine Paradigm}

Many evolutionary techniques for solving different types of problems are developed and inspired from nature (as: immune systems, ant colony, hereditary principles, and so on). Our proposed algorithm for multiobjective optimization applies several principles inspired of the endocrine system. Even if the endocrine system has a particular dependence of nervous system, due to the intrinsic mechanisms of control and its functions, it represents by itself a suitable paradigm in the evolutionary computation's landscape.

The endocrine system, through its function and anatomy is relevant as a complex and adaptable system. That is why an artificial shaping of this one seems to be suitable for solving some complex problems.

A quick view on endocrine system reveals how complex and ingenious nature can be. The endocrine system is represented by a collection of glands, which produce chemical messengers called hormones. These signals get through the blood system to the target organs, which have cells containing the appropriate receptors. The receptors of the cells recognize and tie one type of hormone. The hormones provoke profound changes at the level of the target cells. Every type of hormone has a specific shape, recognized only by the target cells. The main glands of the endocrine system are: the pituitary gland (hypophysis), the thyroid gland, the pancreas, the gonads, the adrenal glands and the pineal gland. The pituitary gland is considered the "master gland" of the body. This description is based on the control, which this one exercises.

At the pituitary gland level are produced those hormones which influence and control the cells and the process of the organism. Its role of supervisor is offered in reality by the function of the hypothalamus, this one representing the bound between the nervous and endocrine systems. The hypothalamic neurons secrete hormones that regulate the release of the hormones from the pituitary gland. The hypothalamic hormones are of two types: releasing and inhibiting hormones, reflecting the influence, which they have over the producing of pituitary hormones, called tropes.

In order to better understand the self-control process of the endocrine system, the following example is taken into consideration: the releasing thyroidian hormone (TRH) produced by the hypothalamus actions on the hypophysis determining the secretion of the thyroidian stimulation hormone (TSH). This one will act on the target organ i.e. thyroid gland. The action of the TSH hormone on the thyroid results the secretion of the specific thyroidian hormones. Thus, when the concentration of the specific thyroidian hormones becomes too high or too low, through a negative feedback process, the hypothalamus is announced about this aspect. So, using inhibiting or releasing hormones the hypothalamus acts on the hypophysis producing an inhibition/releasing in the production of the thyroidian stimulation hormones (TSH) with a direct effect on the thyroid gland activity and thus, a coming back to normal of the thyroidian hormones concentration.

Concluding, the concentration of specific hormones is controlled through a feedback mechanism. So, a special type of hormones generated by hypophysis, called tropes, have the role to supervise and to imprint the releasing or inhibiting of specific hormones. This principle is adopted in our algorithm for the diversity preservation. 
Simplifying, the endocrine system's mechanism for controlling the hormonal concentration is shown in the next figure.

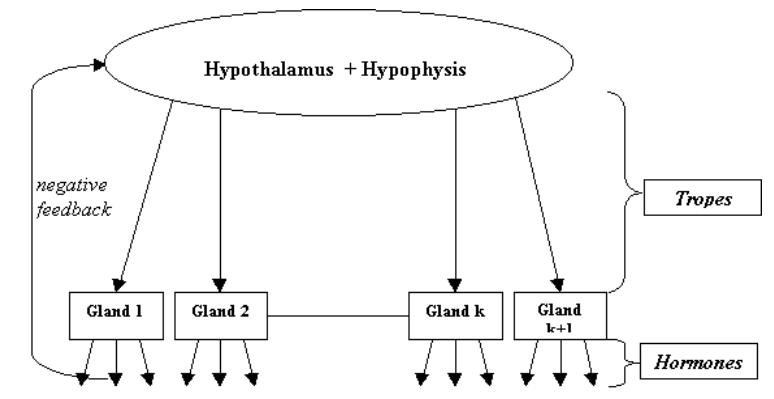

Fig. 1. Control scheme of endocrine system

\section{MENDA Technique}

The principle of the proposed method relies on keeping two populations: an active population of hormones, $H_{t}$, and a passive population of non-dominated solutions, $A_{t}$. The members of the passive population behave as a population of elite and also have a supplementary function: to lead the hormones toward the Pareto front, keeping them as much as possible well distributed among the search space. These two populations correspond to the two classes of hormones in endocrine paradigm:

1. Specific hormones, which are released by different glands of the body - the active population $H_{t}$.

2. The hormones of control (tropes), which are produced by control level of the endocrine system (hypothalamus and hypophysis) in order to supervise the density of each type of hormone - the passive population $A_{t}$.

The population of control, $A_{t}$, is modified at each generation. The new population $A_{t+1}$ gathers all non-dominated solutions from the population $U_{t}$, which has resulted from merging current population of hormones $H_{t}$ and the previous population $A_{t}$. This manner of changing the population of controllers assures us that the non-dominated solutions from the previous population $A_{t}$ cannot be lost if they still remain nondominated after the active population $H_{t}$ has changed.

The passive population, $A_{t}$, doesn't suffer modification at the individual's level, under the variation operators as crossover and mutation. It behaves as an elite population of non-dominated solutions from the current generation, which is only actualized at each generation. Finally, the population $A_{t}$ contains a predetermined number of non-dominated vectors and provides a good approximation of the Pareto front.

At each generation $t$, the members of $H_{t}$ are classified into $s a$ classes. A corresponding controller from $A_{t}$ supervises a particular class. The idea is that each hormone $h$ from $H_{t}$ is supervised by the nearest controller $a_{i}$ from $A_{t}$ : 
An Evolutionary Technique for Multicriterial Optimization Based on Endocrine Paradigm 5

$$
C\left(a_{i}\right)=\left\{h \in H_{t}, \operatorname{dist}\left(h, a_{i}\right)=\min \left(\operatorname{dist}\left(h, a_{j}\right), \mathrm{j}=1,2, \ldots, s a\right)\right\}
$$

Each member $a$ from the set $A_{t}$ has a similar control function as a trop from endocrine paradigm.

Another specific issue of the proposed algorithm is the manner of selecting and recombining the hormones in order to generate descendants.

Among the members of the current population $H_{t}$ a special kind of sharing results. Due to the fact that each individual $a_{i}$ from population $A_{t}$ controls a class of hormones: $C\left(a_{i}\right)$, this individual of control, $a_{i}$, imprints to each hormone a probability of selecting it as first parent. The selection of the first parent is made proportionally to the value of its class, which is calculated according to the next formula:

$$
\operatorname{val}_{i}=1 / \text { sizeof }\left(C\left(a_{i}\right)\right)
$$

The first parent is selected from the population $H_{t}$, proportionally with value of its class; so, we can affirm that the hormones from a particular class locally share the resources. By this manner of selecting first parent, the less crowded hormones are preferred and those zones from the search space, which are apparently disappearing, would be revived.

Let $h_{k}$ be the selected first parent and $a_{i}$, its controller. The second parent $h_{l}$, the mate of the parent $h_{k}$, is selected only from the $h_{k}$ 's class, $C\left(a_{i}\right)$. Parent $h_{l}$ is proportionally selected with its performance, where performance value of a hormone $h$ is given by the next formula, where $n r$ dominated - represents the number of solutions from $H_{t}$, which are dominated by the hormone $h$ :

$$
\text { performance }(h)=n r \_ \text {dominated } / s h
$$

By selecting the second parent proportionally with its performance, the method assures a faster convergence of the population toward the Pareto front.

Crossover operator recombines two parents and produces a single descendant, which is accepted into the next population.

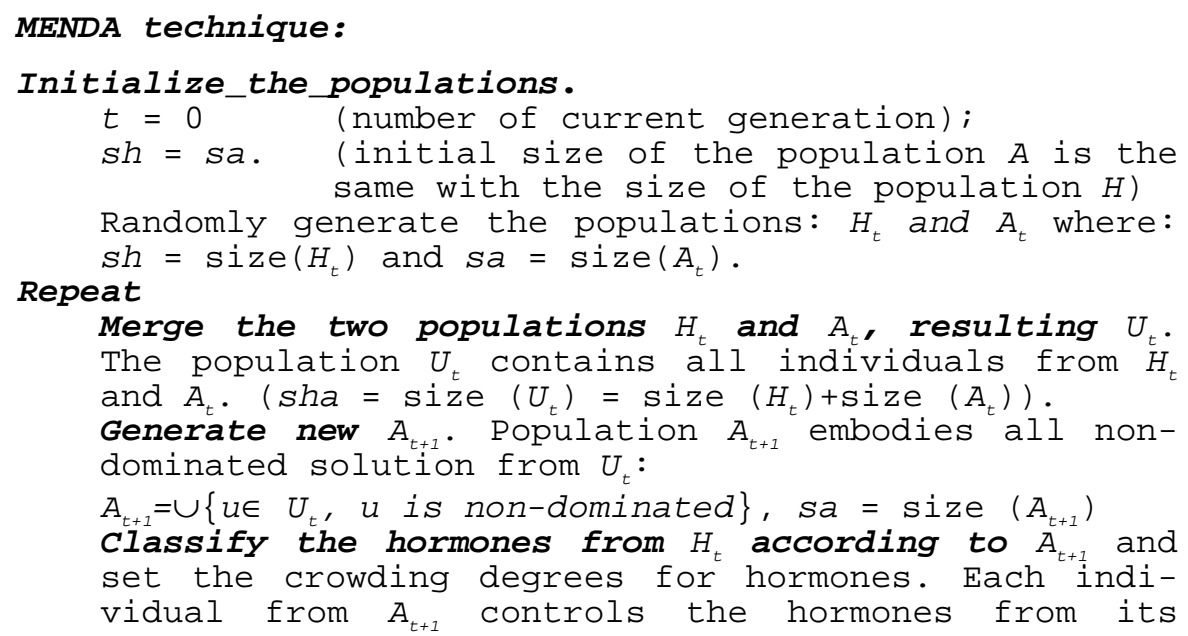




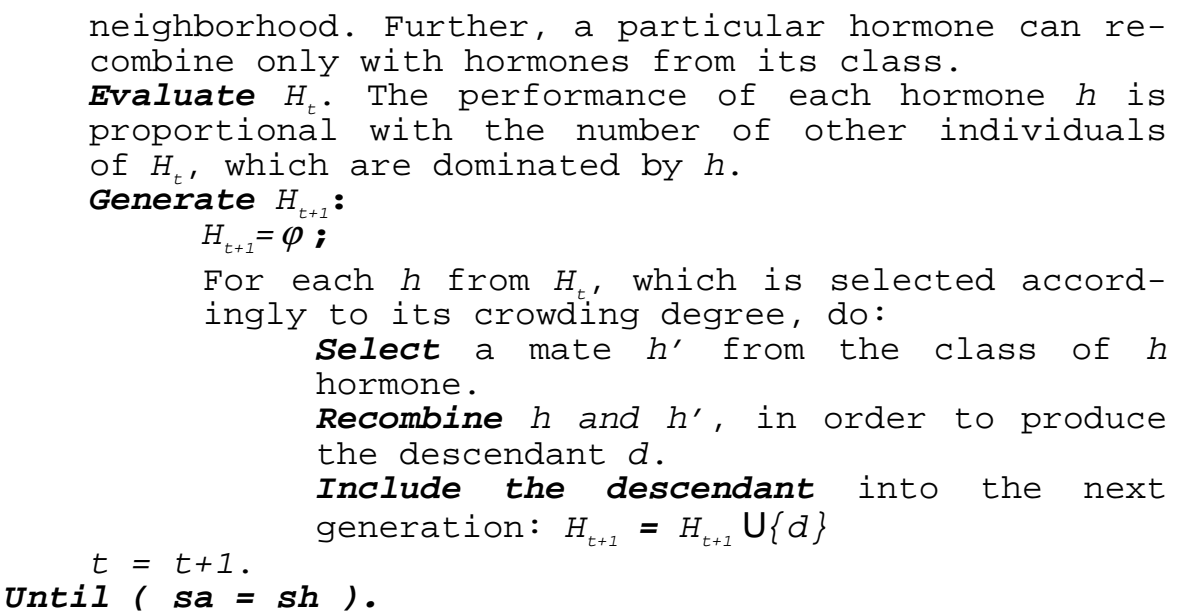

Remarks: The algorithm ends when all individuals from $H_{t}$ became non-dominated. Condition $(s a=s h)$ could be replaced with another condition, as attaining a prefixed number of generations. Numerical experiments proved that for a population of size 100 , the condition $(s a=s h)$ is satisfactory for detecting an approximate Pareto front.

We also noticed that less crowded individuals or those individuals, which are distant from the set $A$, have a significant role for diversity preservation. So, the set $A$ could also embody those individuals.

\section{Results}

We present in this paper several results obtained with a popular difficult test suit (ZDT 1-6 bi-objective problems). [2]. $F$ is a bi-objective function: $F(\bar{x})=\left(f_{1}(\bar{x}), f_{2}(\bar{x})\right)$ and the optimization problem is:

$$
\left\{\begin{array}{l}
\text { Minimize } F(x) \text {, } \\
\text { subject to } f_{2}(x)=g\left(x_{2}, \ldots, x_{m}\right) h\left(f_{1}\left(x_{1}\right), g\left(x_{2}, \ldots x_{m}\right)\right. \\
\text { where } \quad x=\left(x_{1}, \ldots, x_{m}\right)
\end{array}\right.
$$

Table 1. ZDT test problems for multicriterial optimization are described. The function F5 is omitted in the following test results.

\begin{tabular}{|l|l|l|l|}
\hline$F_{1}$ & $f_{1}\left(x_{1}\right)=x_{1}, g\left(x_{2}, \ldots, x_{m}\right)=1+9 \cdot \sum_{i=2}^{m} \frac{x_{i}}{m-1}$, & $\begin{array}{l}x_{i} \in[0,1], \\
i=1,2, \ldots, m \\
m=30\end{array}$ & $\begin{array}{l}\text { Pareto front } \\
\text { is formed } \\
\text { by } g(x)=1\end{array}$ \\
\hline$F_{2}$ & $f_{1}\left(x_{1}\right)=x_{1}, g\left(x_{2}, \ldots, x_{m}\right)=1+9 \cdot \sum_{i=2}^{m} \frac{x_{i}}{m-1}$, & $\begin{array}{l}x_{i} \in[0,1], \\
i=1,2, \ldots, m\end{array}$ & $\begin{array}{l}\text { Pareto front } \\
\text { is formed } \\
\text { by } g(x)=1\end{array}$ \\
\hline
\end{tabular}


An Evolutionary Technique for Multicriterial Optimization Based on Endocrine Paradigm 7

\begin{tabular}{|c|c|c|c|}
\hline & $h\left(f_{1}, g\right)=1-\left(f_{1} / g\right)^{2}$ & $m=30$ & \\
\hline$F_{3}$ & $\begin{array}{l}f_{1}\left(x_{1}\right)=x_{1}, g\left(x_{2}, \ldots, x_{m}\right)=1+9 \cdot \sum_{i=2}^{m} \frac{x_{i}}{m-1} \\
h\left(f_{1}, g\right)=1-\sqrt{f_{1} / g}-\left(f_{1} / g\right) \sin \left(4 \pi f_{1}\right)\end{array}$ & $\begin{array}{l}x_{i} \in[0,1] \\
i=1,2, \ldots, m \\
m=30\end{array}$ & $\begin{array}{l}\text { Pareto front } \\
\text { is formed } \\
\text { by } g(x)=1\end{array}$ \\
\hline$F_{4}$ & $\begin{array}{l}f_{1}\left(x_{1}\right)=x_{1} \\
g\left(x_{2}, \ldots, x_{m}\right)=1+10(m-1)+\sum_{i=2}^{m}\left(x_{i}-10 \cos \left(4 \pi x_{i}\right)\right) \\
h\left(f_{1}, g\right)=1-\sqrt{f_{1} / g}\end{array}$ & $\begin{array}{l}x_{1} \in[0,1] \\
x_{i} \in[-5,5] \\
i=2,3, \ldots, m \\
m=10\end{array}$ & $\begin{array}{l}\text { Pareto op- } \\
\text { timal front } \\
\text { is formed } \\
\text { by } g(x)=1 . \\
\text { The best } \\
\text { Pareto front: } \\
g(x)=1.25 \text {. }\end{array}$ \\
\hline$\overline{F_{6}}$ & $\begin{array}{l}f_{1}\left(x_{1}\right)=1-\exp \left(-4 x_{1}\right) \sin ^{6}\left(6 \pi x_{1}\right) \\
g\left(x_{2}, \ldots, x_{m}\right)=1+9 \cdot\left(\sum_{i=2}^{m} \frac{x_{i}}{m-1}\right)^{1 / 4} \\
h\left(f_{1}, g\right)=1-\left(f_{1} / g\right)^{2}\end{array}$ & $\begin{array}{l}x_{i} \in[0,1] \\
i=1,2, \ldots, m \\
m=10\end{array}$ & $\begin{array}{l}\text { Pareto op- } \\
\text { timal front } \\
\text { is formed } \\
\text { by } g(x)=1\end{array}$ \\
\hline
\end{tabular}

A specific characteristic of those functions made the population to converge toward a segment of the Pareto front, a segment that corresponded with the lowest values of the first function. It can be noticed that if the first objective function depends only of one variable (for example: $f_{1}(\bar{x})=x_{1}$ ), the second objective represents a function with $m-1$ variables, where $m$ is usually 30 .

We observed that for $m=2$, the Pareto front is detected using our standard algorithm. Intuitively, we assume that the proposed evolutionary algorithm minimizes the values of the first function more quickly than the values of the second one. Thus, the final solutions correspond to a small part of the Pareto front, where the first objective's values are lower. Due to this fact, a modification must be made in order to obtain the entire Pareto front. Our proposal is to change the variation operator, respectively the crossover operator.

The standard convex crossover operator is applied on two selected parents $x=\left(x_{1}, x_{2}, \ldots, x_{m}\right)$ and $y=\left(y_{1}, y_{2}, \ldots, y_{m}\right)$, resulting the unique descendant $z=\left(z_{1}, z_{2}, \ldots, z_{m}\right)$, where: $z_{i}=(1-q) \cdot x_{i}+q \cdot y_{i}$ and $q$ is randomly generated.

The modified operator generates the unique descendant $z=\left(z_{1}, z_{2}, \ldots, z_{m}\right)$, too. Considering $\lambda$ from $[0,1]$ (usually 0.5 ), representing a parameter of the algorithm, the modified crossover operator performs as follows:

Modified Crossover Operator:

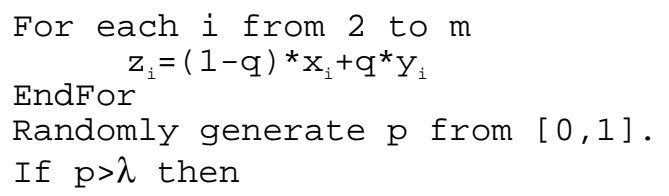




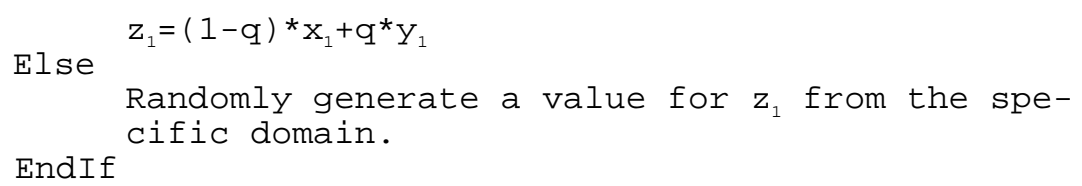

We used the following metrics of performance:

Error Ratio - proposed by Van Veldhuizen; this metric measures the percentage of solutions that are not members of the true Pareto front [5], where $n$ is the number of non-dominated solutions found and:

$$
\begin{gathered}
E R=\frac{\sum_{i=1}^{n} e_{i}}{n} \\
e_{i}=\left\{\begin{array}{c}
0, \text { if vector } i \text { is member of the optimal Pareto front } \\
1, \text { otherwise }
\end{array}\right.
\end{gathered}
$$

Spacing - proposed by Schott; this metric measures the distance variance of the neighboring vectors among the Pareto front [6], where $n$ is the number of nondominated solutions found, $\bar{d}$ is the average of all $d_{i}$ and:

$$
\begin{gathered}
S=\sqrt{\frac{1}{n-1} \sum_{i=1}^{n}\left(\bar{d}-d_{i}\right)^{2}} \\
d_{i}=\min _{j}\left(\left|f_{1}^{i}(x)-f_{1}^{j}(x)\right|+\left|f_{2}^{i}(x)-f_{2}^{j}(x)\right|\right), i, j=1, \ldots, n
\end{gathered}
$$

We also count the number of non-dominated solutions found and the number of generations produced.

MENDA algorithm is performed for 20 times. For each run, the following values are kept: the number of generations produced, the number of non-dominated solutions, the value of Spacing metric for the non-dominated front, and the error ration of the final front.

The table 2 shows the average values per run of these metrics and the best value obtained for each metric. In our tests, the algorithm stops when the error ration became zero.

The results indicated next were found using the following parameters: the size of the active population $H$ is 100 and the passive population $A$ contains maximum 100 members. The supplementary parameter $\varepsilon$ is useful to estimate if two non-dominated solutions are clearly distinct or not: $\varepsilon=10^{-2}$.

First parent is selected by using the tournament selection operator and the second parent is selected among the neighborhood of the first one, by using the proportional selection.

Next, the visual representations of the results are shown: 
An Evolutionary Technique for Multicriterial Optimization Based on Endocrine Paradigm 9
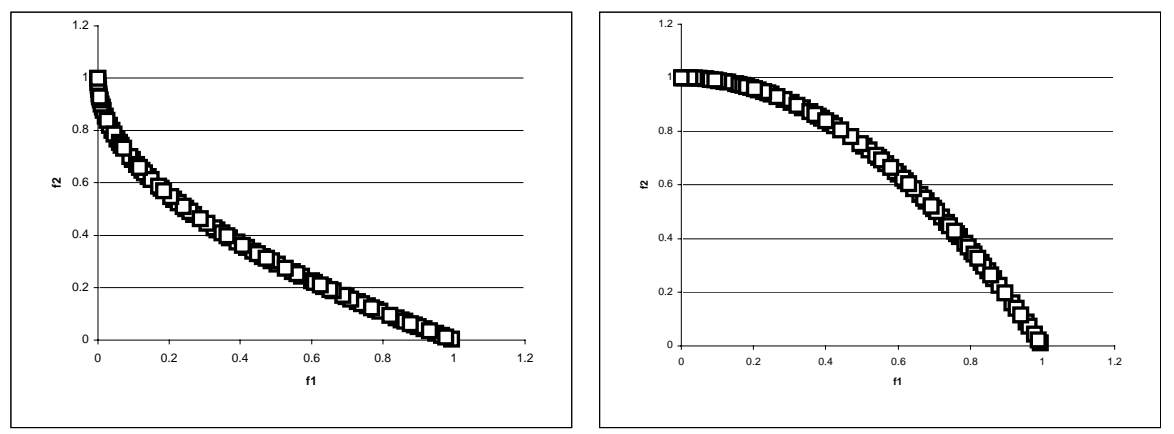

Fig. 2. Visual representation of the non-dominated solutions found for test functions F1 and F2. The figure present the final population of tropes (non-dominated solutions) obtained from a single run of the algorithm.
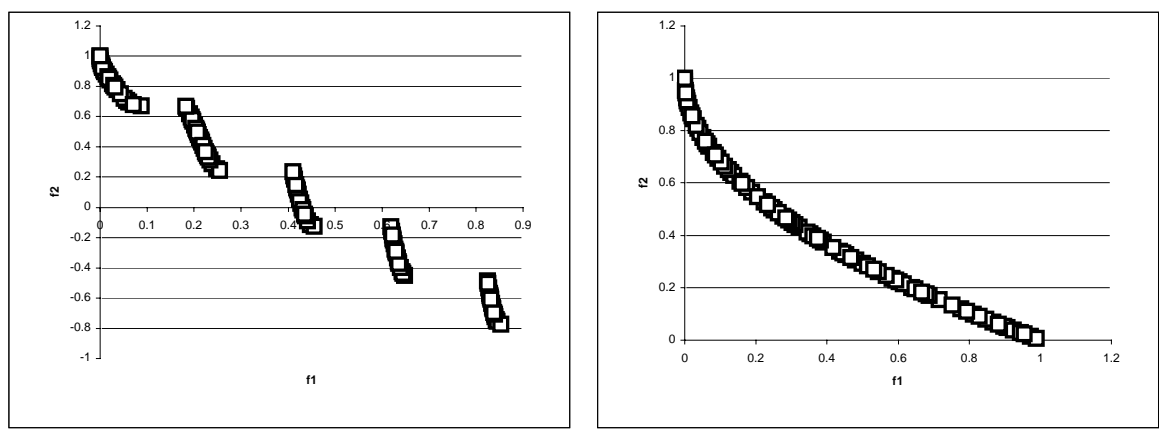

Fig. 3. Visual representation of the final populations of non-dominated solutions obtained in a single run of the MENDA algorithm for the test functions F3 and F4.

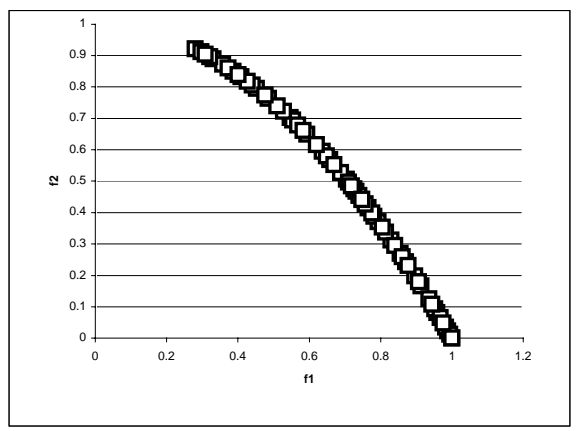

Fig. 4. Visual representation of the non-dominated solutions found for the test functions F6. 
Table 2. The average values per run of the Spacing and Error Ratio metrics and the best value obtained for each metric. Also, the average and the best values of the number of non-dominated solutions obtained in a run, respectively, the number of generations produced, are indicated.

\begin{tabular}{|c|c|c|c|c|c|}
\hline & & $\begin{array}{c}\text { Number of } \\
\text { Generations }\end{array}$ & $\begin{array}{c}\text { Non-dominated } \\
\text { solutions }\end{array}$ & SPACING & $\begin{array}{l}\text { ERROR } \\
\text { RATIO }\end{array}$ \\
\hline \multirow{3}{*}{$F 1$} & Average & 60 & 83 & 0.0056 & 0.00 \\
\hline & Best & 54 & 88 & 0.0046 & 0.00 \\
\hline & Worst & 68 & 77 & 0.0069 & 0.00 \\
\hline \multirow{3}{*}{$F 2$} & Average & 65 & 83 & 0.0058 & 0.00 \\
\hline & Best & 54 & 90 & 0.0041 & 0.00 \\
\hline & Worst & 88 & 75 & 0.0079 & 0.00 \\
\hline \multirow{3}{*}{$F 3$} & Average & 121 & 97 & 0.0061 & 0.00 \\
\hline & Best & 86 & 100 & 0.0047 & 0.00 \\
\hline & Worst & 162 & 90 & 0.0072 & 0.00 \\
\hline \multirow{3}{*}{ F4 } & Average & 37 & 82 & 0.0059 & 0.00 \\
\hline & Best & 32 & 88 & 0.0043 & 0.00 \\
\hline & Worst & 43 & 78 & 0.0080 & 0.00 \\
\hline \multirow{3}{*}{ F6 } & Average & 145 & 65 & 0.0059 & 0.00 \\
\hline & Best & 122 & 70 & 0.0046 & 0.00 \\
\hline & Worst & 251 & 60 & 0.0087 & 0.00 \\
\hline
\end{tabular}

We choose to make a visual comparison of the results produced by MENDA algorithm against the results obtained with two popular techniques: SPEA and NSGA. We plotted in the following graphs the nondominated vectors produced in a single run of each algorithm. The experimental results regarding the evolutionary algorithms NSGA and SPEA were found on the web page

http://www.tik.ee.ethz.ch/ zitzler/testdata.html/\#testproblems.

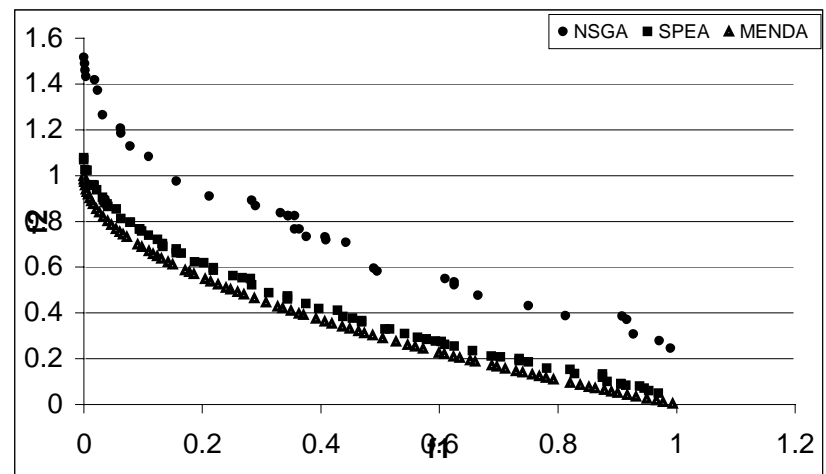

Fig. 5. MENDA versus SPEA and NSGA for the test functions F1. 
An Evolutionary Technique for Multicriterial Optimization Based on Endocrine Paradigm 11

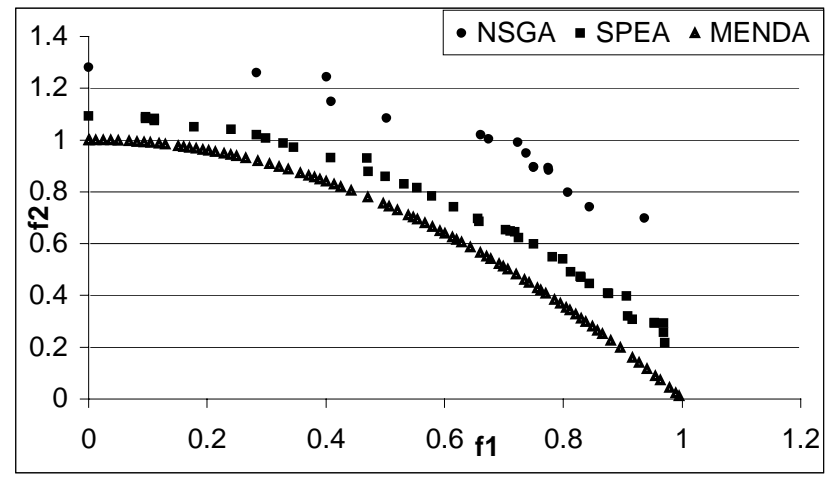

Fig. 6. MENDA versus SPEA and NSGA for the test functions F2.

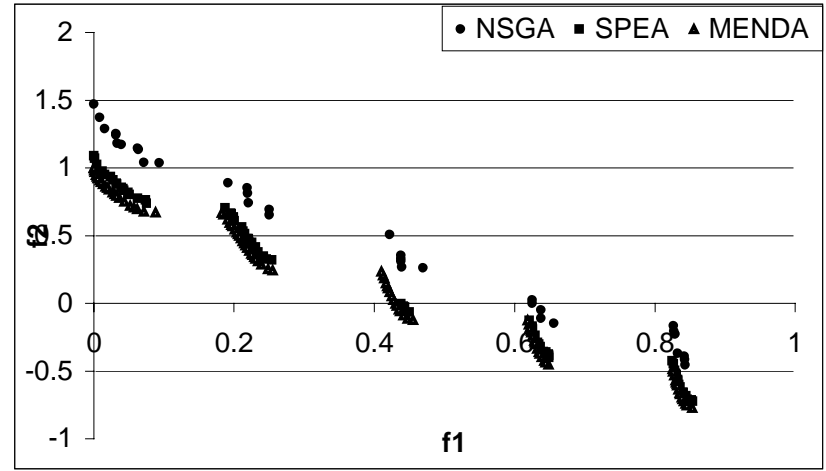

Fig. 7. MENDA versus SPEA and NSGA for the test functions F3.

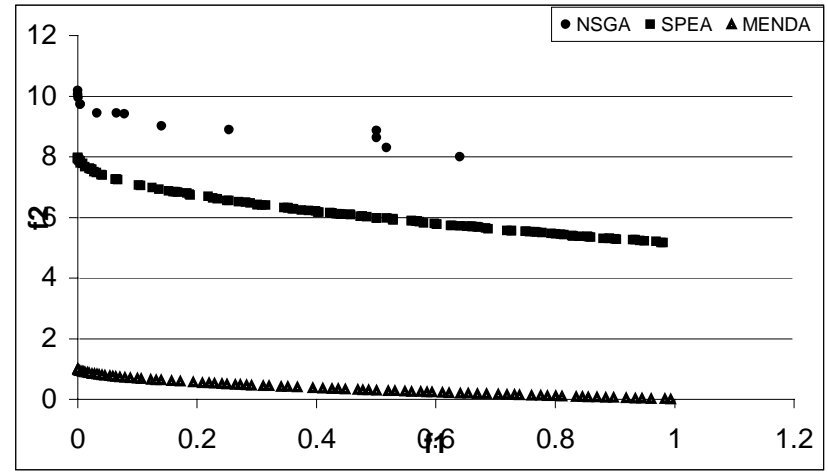

Fig. 8. MENDA versus SPEA and NSGA for the test functions F4. 


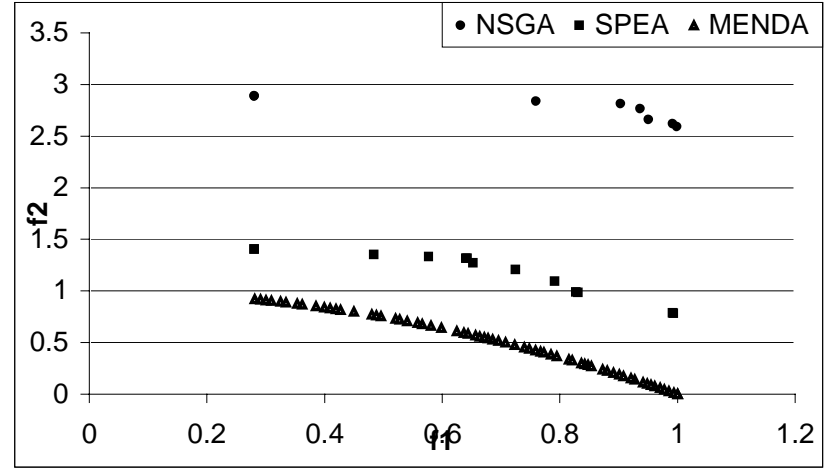

Fig. 9. MENDA versus SPEA and NSGA for the test functions F6

\section{Conclusions}

Nature offers complex phenomena, processes and systems, which can inspire us in many ways. An example of this kind is the natural endocrine system. It reveals us as a dynamic, adaptive and semi-independent system. Its complex structural and functional features suggest us a possible imitation of its characteristics for developing new techniques for multiobjective optimization. It is clear that hormonal system was ignored by now. Our work essentially attempts to mimic hormones' behavior in order to solve difficult problems like multiobjective optimization. Consequently, we propose a new technique based on endocrine paradigm, called MENDA (Multiobjective Endocrine $\boldsymbol{A}$ lgorithm). It provides satisfactory solutions in our tests.

\section{References}

1. Coello C. A.. C. (1999), An Updated Survey of Evolutionary Multiobjective Optimization Techniques : State of the Art and Future Trends, In 1999 Congress on Evolutionary Computation, Washington, D.C..

2. Deb, K. (1999), Multi-objective genetic algorithms: Problem difficulties and construction of test functions, Evolutionary Computation, 7(3).

3. Dumitrescu D., Lazzerini B., Jain L.C., Dumitrescu A. (2000), Evolutionary Computation, CRC Press, Boca Raton London, New York, Washington D.C.

4. Fonseca, C.M., Fleming, P.J. (1995), Multiobjective Optimization and Multiple Constraint Handling with Evolutionary Algorithms I: A Unified Formula, Research Report no. 564, Sheffield, United Kingdom.

5. Goldberg D.E., Genetic Algorithms in Search, Optimization, and Machine Learning, Addison-Wesley Publishing Company, Inc., 1989.

6. Laumanns, M., Thiele, L., Zitzler, E., Deb, K., On the Convergence and Diversity Preservation Properties of Multi-Objective Evolutionary Algorithms, TIK - Report no. 108, Switzerland, 2001. 